\title{
Practical Aspects of Acoustic Leaky-Wave Antennas Applied to Underwater Direction Finding ${ }^{\dagger}$
}

\author{
Ivan Felis-Enguix ${ }^{1, *}$, Jorge Otero-Vega ${ }^{2}$, María Campo-Valera ${ }^{3}$, I. Villó-Pérez ${ }^{3}$ and J. L. Gómez- \\ Tornero ${ }^{4}$ \\ 1 Centro Tecnológico Naval y del Mar (CTN), Fuente Álamo, 30320 Murcia, Spain \\ 2 Institut d'Investigació per a la Gestió Integrada de les Zones Costaneres (IGIC), Universitat Politècnica de \\ València (UPV), Gandia, 46730 València, Spain; jorotve@upv.es \\ 3 Departamento de Electrónica, Tecnología de Computadores y Proyectos, Universidad Politécnica de \\ València (UPCT), Cartagena, 30202 Murcia, Spain; isidro.villo@upct.es \\ 4 Departamento de Tecnologías de la Información y las Comunicaciones, Universidad Politécnica de \\ València (UPCT), Cartagena, 30202 Murcia, Spain; josel.gomez@upct.es \\ * Correspondence: ivanfelis@ctnaval.com \\ + Presented at the 7th Electronic Conference on Sensors and Applications, 15-30 November 2020; Available \\ online: https://ecsa-7.sciforum.net/.
}

Published: 15 November 2020

\begin{abstract}
Acoustic leaky-wave antennas (ALWAs) have demonstrated the capacity to steering directive sound waves in frequency-dependent directions, due to the inherent dispersive radiation characteristic of leaky modes [1-3]. Compared to more conventional uniform linear array (ULA) acoustic traducers for electronic beam steering (which rely on multiple sensors), the ALWA allows for single microphone operation. Thus, ALWAs offer a direct mechanism to scan a directive acoustic beam in the angular space by simply sweeping the operating frequency of the acoustic signal [3], which envisions cost-efficient single-transducer direction finders for SONAR applications [4]. In this paper we study for the first time, some important features of an ALWA for acoustic underwater Direction-of-Arrival (DoA) estimation applications. First, we report for the first time on the necessity to shape the radiated ALWA beams in both far- and near-field zones to improve the DoA estimation performance, following similar techniques recently applied for low-cost frequencyscanned direction-finding radars based on LWAs $[5,6]$. Also, we analyse the capacity to reduce the Side Lobe Level (SLL) for enhanced performance [3], demonstrating aperture tapering techniques [7] to the ALWA for the first time. These aspects are of much interest for real applications of ALWA in innovative SONAR systems for underwater scenarios.
\end{abstract}

Keywords: Acoustic Leaky-Wave Antenna; sonar; direction finding; underwater

\section{Introduction}

In acoustic radiation phenomena, part of the energy is gradually lost in the form of radiation toward the external boundaries of an open region, reducing the amplitude of oscillations even when the system is ideally no dissipative [8]. This phenomenon is widely used in various applications where it is desired to improve the directivity in order to make the signal transmission more secure and efficient, as in the case of Acoustic Leaky-Wave Antenna (ALWA). The basic design for a ALWA is a one- or two-dimensional waveguide with a slit or array of shunts where the leaking apertures can be used to control radiated directionality as a function of frequency [3]. Despite of the use of electromagnetic leaky-wave antenna in the electromagnetic field has been widely studied analytically, experimentally and used in various directive radiation applications [9-11], the 
properties and performances of ALWA have not yet been studied in depth and are being studied in recent years [3-5].

In most of the studied cases, the applications focus on the pressure distribution in air purposes where geometries designed for directive patterns are shown in a bandwidth that depends on the propagation medium. In this work, results of finite element simulation models (FEM) for open underwater media are presented. In this line, it is studied the influence of geometric changes in the device. For these propose, the studies start from an underwater ALWA reference geometry (with a starting frequency of about $2500 \mathrm{~Hz}$ and scanning capacity of $60^{\circ}$ ) and the behaviour of the directivity, SPL (Sound Pressure Level) and SLL is tested, from different modifications in its geometric characteristics.

\section{Theoretical Framework}

An acoustic leaky-wave antennas (ALWA) can be defined as an open acoustic waveguide of length $L$ which propagates a leaky mode. Leaky modes are complex modes which continuously radiate to the outer space as they propagate through the open waveguide. Apart from a phase constant $\beta[\mathrm{rad} / \mathrm{m}]$, the complex propagation wavenumber of leaky modes accounts for the longitudinal attenuation rate due to radiation, given by the leakage constant $\alpha[\mathrm{nep} / \mathrm{m}]$ :

$$
k=\beta-j \alpha\left(m^{-1}\right)
$$

The acoustic pressure radiation pattern of this ALWAs is in the form of a directive scanned beam, which half-power beam width $(\mathrm{HPBW}, \Delta \theta)$ and scanning direction $\theta_{\mathrm{RAD}}$, are given by:

$$
\begin{gathered}
\Delta \theta \approx \frac{63^{\circ}}{\frac{L}{\lambda} \cos \theta_{R A D}} \\
\theta_{R A D} \approx \operatorname{asin} \frac{\beta}{k_{0}}
\end{gathered}
$$

where $\lambda$ is the sound wavelength and $\mathrm{k}_{0}$ is the free-space wavenumber in the medium surrounding the ALWA (which can be air or water, for instance). One important feature of ALWAs is that, since the leaky mode phase constant is dispersive with frequency $\beta=\beta(f)$, the resulting scanning angle is also frequency dependent. As a result, ALWAs inherently provide frequency-scanning of a directive beam

In the previous studies analysed, it was proposed the use of a frequency-beam scanning LWA for radio direction finding, by acquiring the power received by a broadband signal, for different frequency components [5]. This can be applied for low-cost localization systems which use a single source [6]. Also, the results can be extended to acoustic waves, as proposed for single-microphone direction finding systems [4]. Also, it was mentioned in [8] the necessity to taper the ALWA to reduce the sidelobe level (SLL) of the radiation pattern, and thus improve the angular estimation performance. The tapering of ALWA consist in the engineering of the leaky-mode complex propagation constant-both the phase constant and the leakage rate in (1) - in order to manipulate the radiated acoustic fields and control the synthesis of the beam pattern [7]. Basically, the control of the scanning angle in (2) allows to choose the scanning directions to be sensed at different frequencies, while the control of the leakage rate allows control on the aperture pressure profile, and thus control on the beam shape (including HPBW in (2) and SLL control). This is illustrated in Error! Reference source not found., which sketches the differences between an ALWA with and without tapering of the aperture sound pressure profile. Error! Reference source not found.a shows the case of an ALWA without tapering of the leakage rate $\alpha$ : as a result the acoustic leaky waves induces an exponential SPL profile in the radiating aperture of the ALWA, which creates a theoretical SLL of $-13 \mathrm{~dB}$. If the pressure level is tapered to avoid diffraction at the edges of the ALWA, the SLL can be reduced below $-20 \mathrm{~dB}$. This is shown in Figure 1 for the case of a cosine-tapered sound pressure profile: clearly the shaping of the radiated sound provides lower SLL, as it can be seen in Figure 1c, and this reduces false echoes and interferences in SONAR systems. 


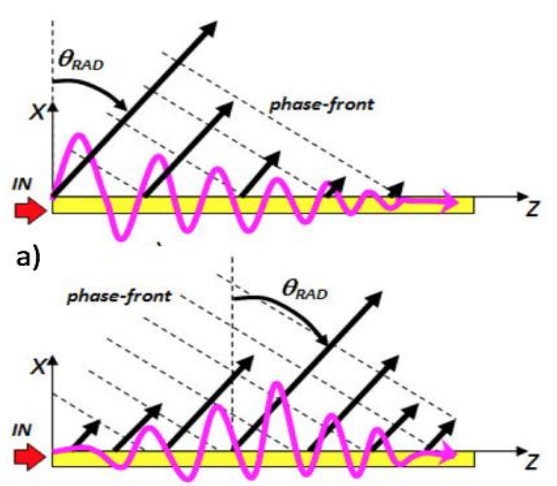

b)

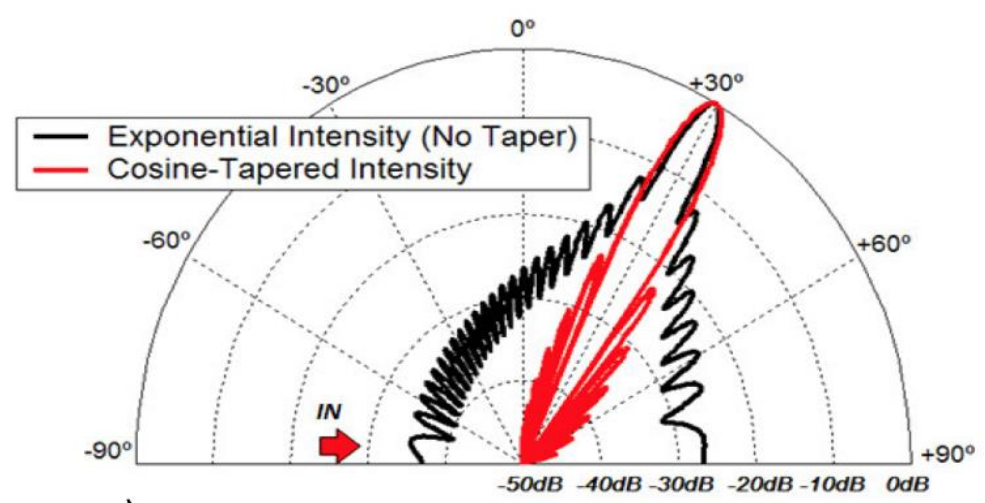

c)

Figure 1. (a) Aperture acoustic leaky fields in a non-tapered leakage level ALWA (exponential pressure profile) (b) The same but for a cosine-tapered pressure profile (c) Corresponding far-field radiation patterns.

In this paper we study the capacity to control the leakage angle and intensity in an ALWA, in order to design tapered ALWAs for improved underwater SONAR acoustic direction-finding systems.

\section{Numerical Modelling and Performance}

The different ALWAs has been modelled with a FEM method (considering its symmetry of revolution) in a frequency depend study. Error! Reference source not found. shows a schematic of the model for a configuration of 22 elements and a cross section showing the simulated elements.

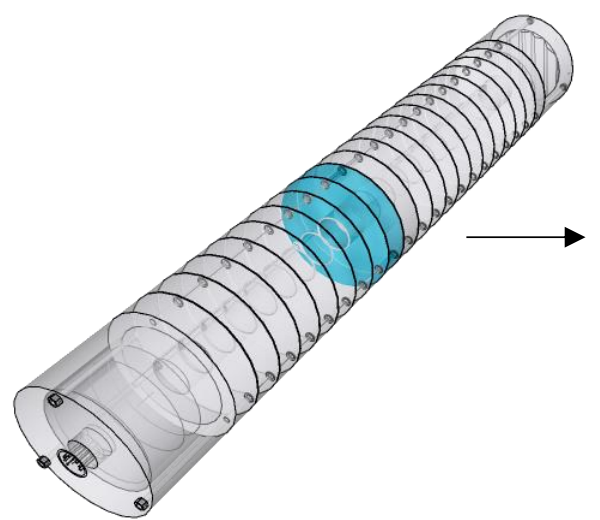

(a)

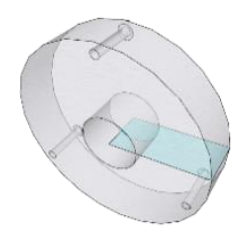

(b)

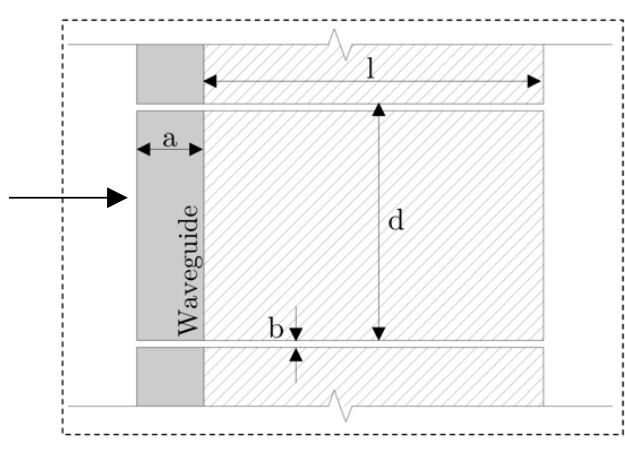

(c)

Figure 3. (a) Geometrical model of the simulated ALWA, (b) detail of one-unit cell and (c) name of the geometric parameters used in this study.

From these simulations, the acoustic field in an open water domain is obtained. From this, different properties of the resulting acoustic radiated field are studied, the Direction of Arrival (DoA), taking into account both the angle and the associated SPL and SLL, both in function of the frequency. Error! Reference source not found.a shows two examples of the acoustic radiated field for two different frequencies of the same ALWA and Error! Reference source not found.b an example of the amplitudes obtained in the far field from which the SLL values are. 


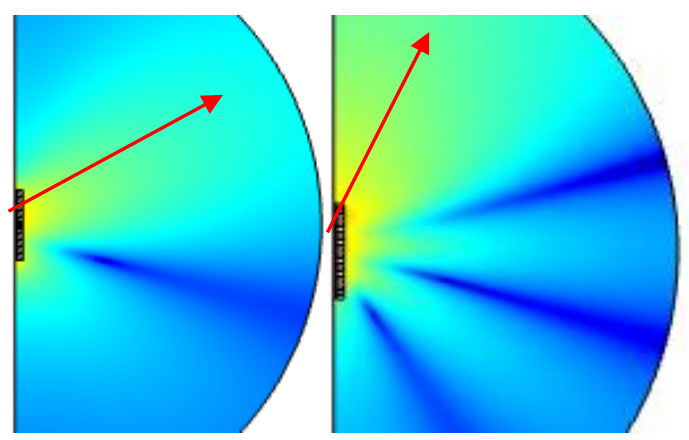

(a)

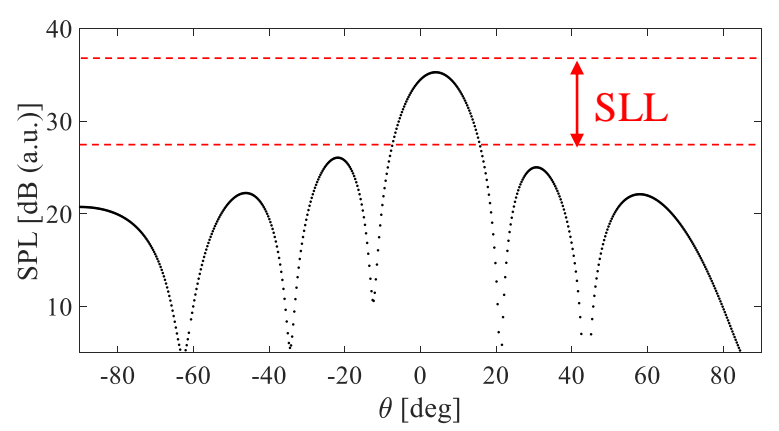

(b)

Figure 4. (a) Acoustic radiated field for two different frequencies of the same ALWA, (b) Sound Pressure Level (SPL) obtained in the far field from which the SLL values are obtained.

\section{Results}

\subsection{Direction-of-Arrival (DoA)}

It is shown the results of the frequency dependence of the radiation angle $(\theta)$ and the amplitude of the main beam as a function of the different geometric parameters $(a, b, d, l)$, the number of unit cells (n) of the ALWA.

- Waveguide inner radius $(a)$. Regarding the radiation angle, by increasing $1 \mathrm{~cm}$ the radius (increase $\mathrm{x} 2$ the radius or, equivalently, $\mathrm{x} 4$ the section area), the lower frequency of radiation reduces from 2500 to $1800 \mathrm{~Hz}$; by reducing $1 \mathrm{~cm}$ the radius (x1/2 the radius or $x 1 / 4$ the section area), this frequency increases to $1800 \mathrm{~Hz}$. Regarding the SPL, as the radius decreases, the radiation levels also decrease following a similar profile, with peaks about $+3 \mathrm{~dB}$ at cut-off frequencies.

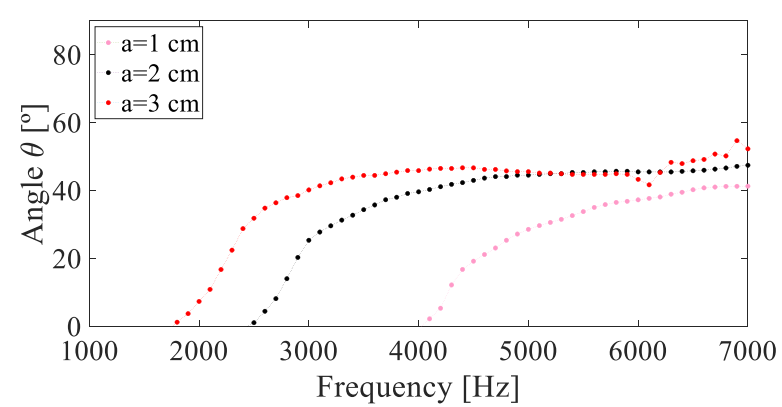

(a)

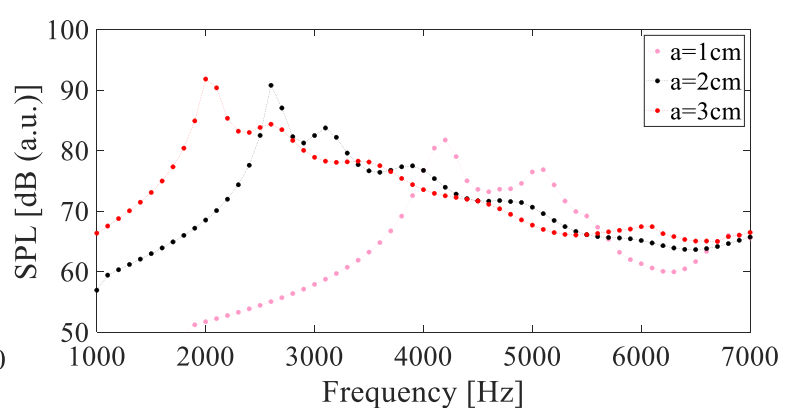

(b)

Figure 5. (a) Frequency dependence of the main radiation angle and (b) sound pressure level, for different waveguide inner radius $(a)$.

- Shunt width (b). By increasing 1 and $2 \mathrm{~mm}$ the shunt width (x2 and $\mathrm{x} 3$ ), the cut-off frequency increases from $2500 \mathrm{~Hz}$ to 3400 and $4000 \mathrm{~Hz}$, respectively, slightly increasing the radiation angle for high frequencies. While the levels of the first radiation peak located at the starting frequency are quite similar, the SPL decreases with frequency more slowly as the parameter $b$ increases. 


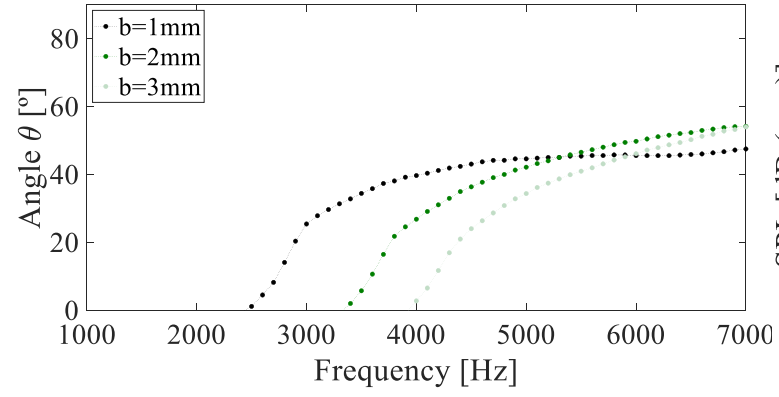

(a)

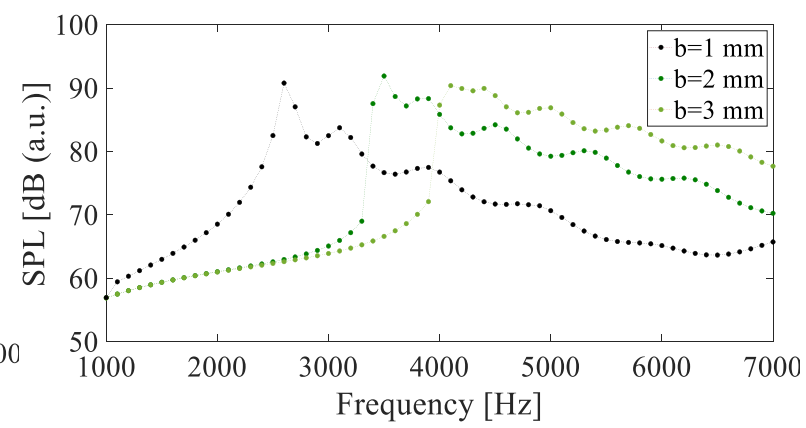

(b)

Figure 6. (a) Frequency dependence of the main radiation angle and (b) sound pressure level, for different shunt widths $(b)$.

- Unit cell length $(d)$ : When increasing the unit cell length from 30 to $50 \mathrm{~mm}(\mathrm{x} 1.7)$, the frequency is slightly reduced from 2500 to $2000 \mathrm{~Hz}$, while when reducing it to $10 \mathrm{~mm}(\mathrm{x} 1 / 3)$, it is considerably increased up to $4600 \mathrm{~Hz}$. Regarding sound pressure levels, the corresponding peaks to the initial radiation frequencies decrease with the unit cell length, while the tails for higher frequencies decrease more rapidly for larger unit cell lengths.

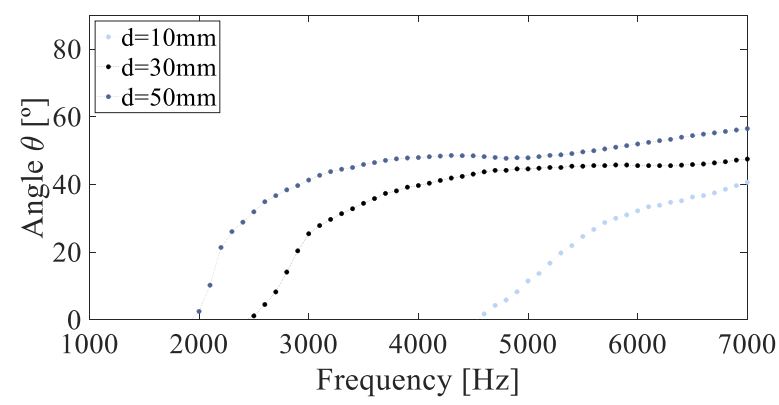

(a)

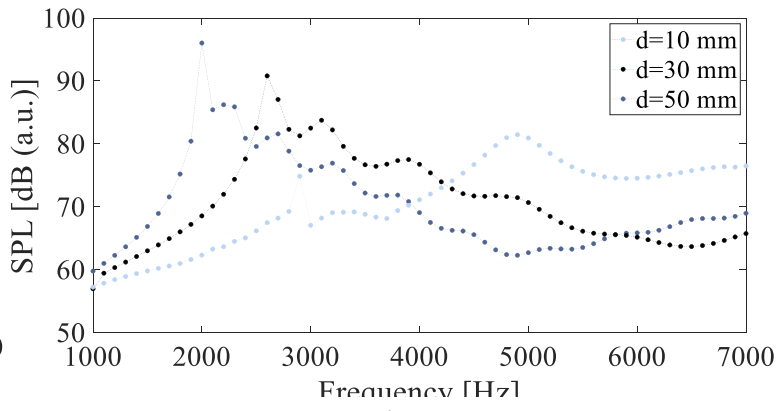

(b)

Figure 7. (a) Frequency dependence of the main radiation angle and (b) sound pressure level, for different unit cell lengths $(d)$.

- Shunt length $(l)$ : For lengths between 1.25 and $10 \mathrm{~cm}$ (one order of magnitude), radiation varies between 2000 and $4000 \mathrm{~Hz}$ (factor two of variation), while maximum radiation angles decreases from 60 to 40 degrees. While the levels of the first radiation peak located at the starting frequency are quite similar (except to $l=10 \mathrm{~cm}$ ), the SPL decreases with frequency more slowly as the parameter $l$ decreases.

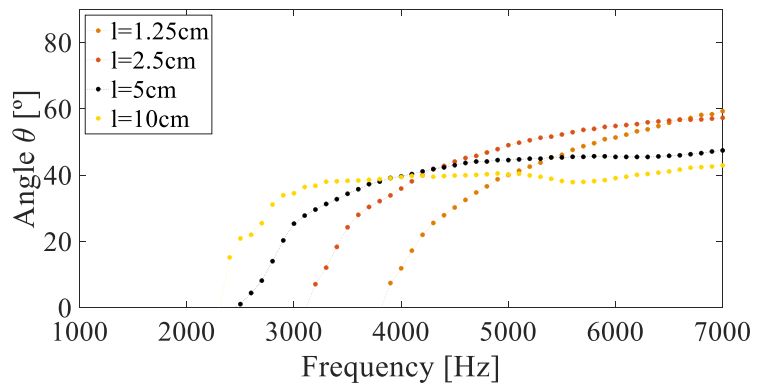

(a)

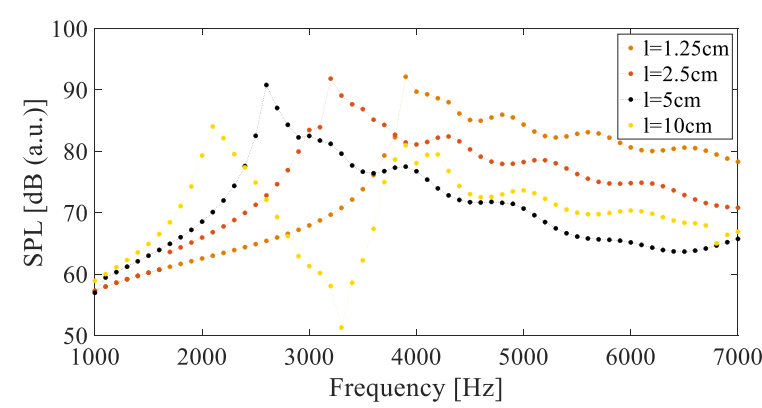

(b)

Figure 8. (a) Frequency dependence of the main radiation angle and (b) sound pressure level, for different shunt lengths $(l)$. 
- Number of unit cells ( $n$ ). It can be seen that, on the one hand, that the lower radiation frequency does not change with the change in the number of cells (this frequency depends only on the geometry) and, on the other hand, when the number of unit cells increases, the radiation angles tend a constant value (between 55 and $60^{\circ}$ ), with little variation for more than 32 unit cells. Regarding SPL, they are similar for high numbers of unit cells, falling by up to $10 \mathrm{~dB}$ between 42 and 12 cells.

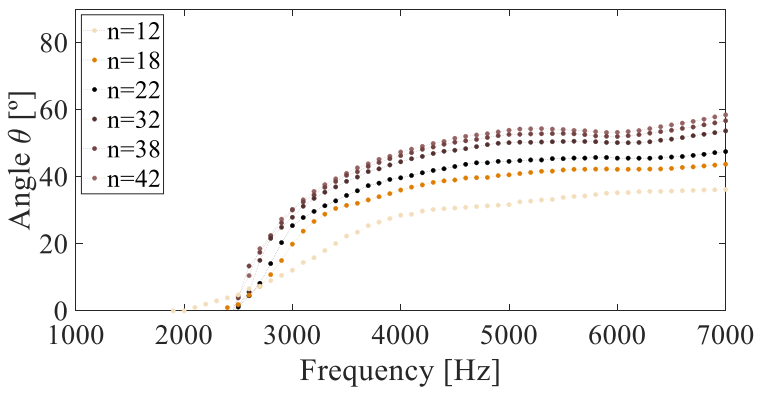

(a)

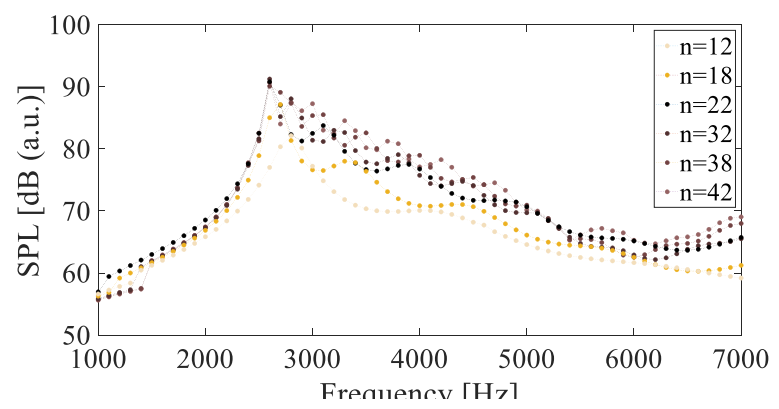

(b)

Figure 9. (a) Frequency dependence of the main radiation angle and (b) sound pressure level, for different numbers unit cells $(n)$.

\subsection{Side Lobe Level (SLL)}

The Error! Reference source not found. show the dependence of the SLL with respect to the main parameters seen above. In all the curves it is observed that the maximum of SLL is obtained for the initial radiation frequency, with values between 15 and $23 \mathrm{~dB}$ re $1 \mu \mathrm{Pa}$ for most of them, except for large $d$ and for high numbers of unit cells $(\mathrm{n}>22)$.

- Waveguide inner radius $(a)$ :

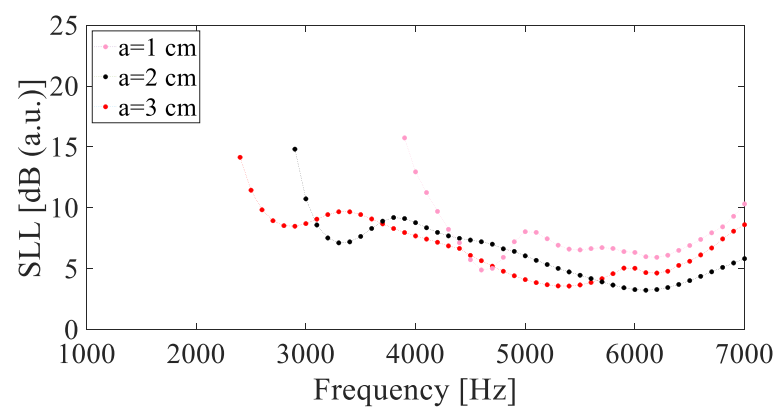

- Unit cell length $(d)$ :

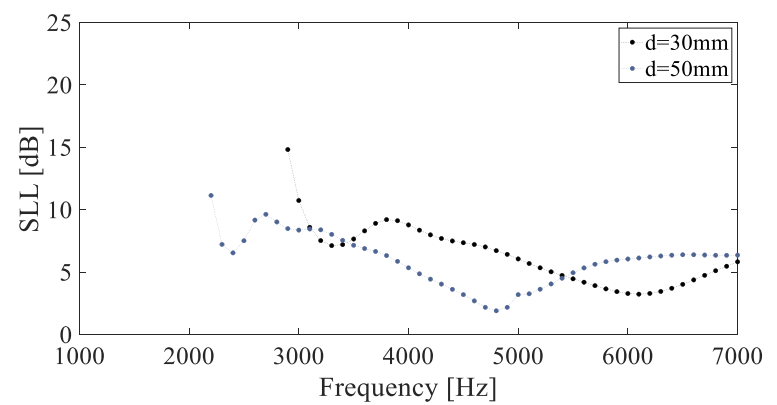

- Shunt width $(b)$ :

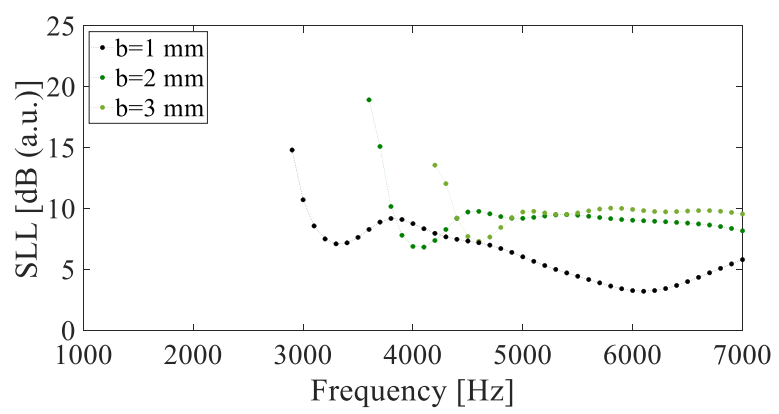

- Number of unit cells $(n)$ :

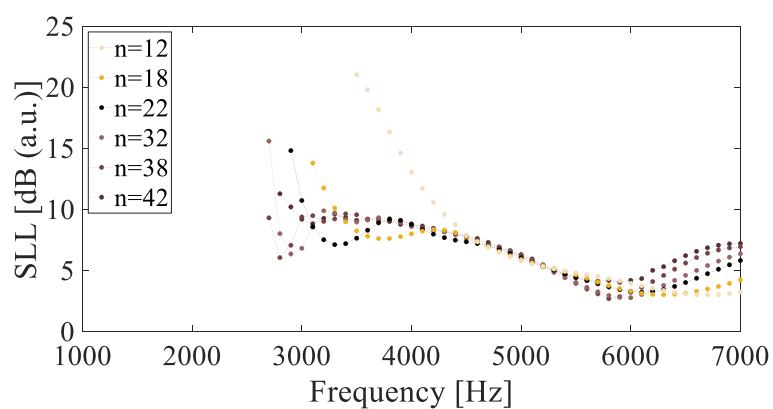

Figure 11. Frequency dependence of the Side Lobe Level (SLL) considering different parameters of the ALWA design. 
Generalizing these results, from the point of view of an improvement of the SLL, it is desired a device with the least number of unit cells $(n)$, with some independence from the waveguide inner radius $(a)$. The parameter $b$ and $d$ can be specified independently.

\section{Conclusions}

For underwater ALWA, as in electromagnetic leaky waveguides, the cylinder cross-section radius " $a$ " is one of the main parameter that determines the cut-off frequency of the propagating mode and this is related to the broadside direction of radiation $\left(\theta=0^{\circ}\right)$, which determines the lower frequency of radiation. In fact, as it can be seen in Error! Reference source not found.a, the cut-off frequency can be tuned from $2500 \mathrm{~Hz}$ to $4000 \mathrm{~Hz}$ as the waveguide inner radius a is stretched from $a=3 \mathrm{~cm}$ to $a=1 \mathrm{~cm}$. Then, as frequency is increased from this cut-off frequency, the radiation angle is scanned from broadside direction $\theta=0^{\circ}$ up to a maximum angle of about $\theta=60^{\circ}$. Also it is common to obtain a leakage level which is higher at this cut-off frequency and drops as frequency is increased and the radiation angle is steered, as it can be seen in the simulations of the SPL in Error! Reference source not found.b. In addition, in the case of ALWA, other geometrical parameters like the shunt width " $b$ " and the unit cell length " $d$ " also strongly affect the cut-off frequency and they can be used in order to tune the desired radiation properties of the devices.

Finally, it can be note that, for the design of a tapered ALWA, the modulation of the waveguide radius " $a$ " allows to control the scanning angle $\theta$ along the antenna longitudinal radiating aperture [7]. This is of crucial interest to synthesize antennas which keep the scanning angle stable along the whole aperture to maximize the directivity, while controlling the pressure intensity distribution to optimize the sidelobe level [8]. Conversely, in other applications the designer prefers to modulate the scanning angle to focus the radiated energy into a near-field focused spot [7].

Author Contributions: Conceptualization, J.L.G.-T. and I.F.-E.; methodology and formal analysis I.F.-E. and J.O.V.; writing - original draft preparation, I.F.-E. and J.O.-V.; writing and editing, I.F.-E. and J.O.-V.; visualization, J.O.-V.; review by all authors; supervision, I.F.-E., J.L.G.-T. All authors have read and agreed to the published version of the manuscript.

Conflicts of Interest: The authors declare no conflict of interest.

\section{References}

1. Naify, C.J.; Layman, C.; Martin, T.P.; Nicholas, M.; Calvo, D.C.; Oriss, G.J. Experimental realization of a variable index transmission line metamaterial as an acoustic leaky-wave antenna. Appl. Phys. Lett. 2013, 102, 20.

2. Esfahlani, S.H.S.; Karkar, S.; Lissek, H. Optimization of an acoustic leaky-wave antenna based on acoustic metamaterial. In Proceedings of the Acoustics 2013, New Delhi, India, 10-15 November 2013.

3. Naify, C.J.; Rogers, J.S.; Guild, M.D.; Rohde, C.A.; Orris, G.J. Evaluation of the resolution of a metamaterial acoustic leaky wave antenna. J. Acoust. Soc. Am. 2016, 139, 6.

4. Esfahlani, H.; Karkar, S.; Lissek, H.; Mosig, J.R. Exploiting the leaky-wave properties of transmission-line metamaterials for single-microphone direction finding. J. Acoust. Soc. Am. 2016, 139, 6.

5. Poveda-García, M.; Cañete-Rebenaque, D.; Gómez-Tornero, J.L. Frequency-Scanned Monopulse Pattern Synthesis Using Leaky-Wave Antennas for Enhanced Power-Based Direction-of-Arrival Estimation. IEEE Trans. Antennas Propag. 2019, 67, 7071-7086.

6. Poveda-García, M.; Gómez-Alcaraz, A.; Cañete-Rebenaque, D.; Martinez-Sala, A.S.; Gómez-Tornero, J.L. RSSI-Based Direction-of-Departure Estimation in Bluetooth Low Energy Using an Array of FrequencySteered Leaky-Wave Antennas. IEEE Access 2020, 8, 9380-9394.

7. Gómez-Tornero, J.L. Unusual tapering of leaky-wave radiators and their applications. In Proceedings of the 5th European Conference on Antennas and Propagation (EUCAP), Rome, Italy, 11-15 April 2011.

8. Naify, C.J.; Woolfe, K.; Layman, C.N.; Rogers, J.S.; Guild, M.D.; Orris, G. Designing beampatterns with tapered leaky wave antennas. J. Acoust. Soc. Am. 2017, 141, 3374.

9. Lim, S.; Caloz, C.; Itoh, T. Metamaterial-based electronically controlled transmission-line structure as a novel leaky-wave antenna with tunable radiation angle and beamwidth. IEEE Trans. Microw. Theory Tech. 2004, 52, 2678-2690. 
10. Jackson, D.R.; Caloz, C.; Itoh, T. Leaky-Wave Antennas. Proc. IEEE 2012, 100, 2194-2206.

11. Liu, J.; Zhou, W.; Long, Y. A Simple Technique for Open-Stopband Suppression in Periodic Leaky-Wave Antennas Using Two Nonidentical Elements Per Unit Cell. IEEE Trans. Antennas Propag. 2018, 66, 27412751.

Publisher's Note: MDPI stays neutral with regard to jurisdictional claims in published maps and institutional affiliations.

(C) 2020 by the authors. Submitted for possible open access publication under the terms and conditions of the Creative Commons Attribution (CC BY) license (http://creativecommons.org/licenses/by/4.0/). 